\title{
Intertheoretic Reduction, Confirmation, and Montague's Syntax-Semantics Relation
}

\author{
Kristina Liefke • Stephan Hartmann
}

Received: date / Accepted: date

\begin{abstract}
Intertheoretic relations are an important topic in the philosophy of science. However, since their classical discussion by Ernest Nagel, such relations have mostly been restricted to relations between pairs of theories in the natural sciences. This paper presents a case study of a new type of intertheoretic relation that is inspired by Montague's analysis of the linguistic syntax-semantics relation. The paper develops a simple model of this relation. To motivate the adoption of our new model, we show that this model extends the scope of application of the Nagelian (or related) models and that it shares the epistemological advantages of the Nagelian model. The latter is achieved in a Bayesian framework.
\end{abstract}

Keywords Intertheoretic relations - Reduction · Montague semantics ·

Syntax-semantics relation · Philosophy of linguistics · Bayesian confirmation

K. Liefke

Institute for Linguistics, Goethe University Frankfurt, Norbert-Wollheim-Platz 1, 60323 Frankfurt am Main, Germany. Tel.: +49-(0)69-798-32052, E-mail: Liefke@lingua.unifrankfurt.de

S. Hartmann

Munich Center for Mathematical Philosophy, Ludwig-Maximilians-University Munich, Geschwister-Scholl-Platz 1, 80539 Munich, Germany. Tel.: +49-(0)89-2180-3320, E-mail: S.Hartmann@lmu.de 


\section{Introduction}

Epistemic relations between pairs of co-existing theories are an important topic in the philosophy of science. These relations involve a connection between the laws (or 'propositions') of two related theories - typically, the derivability of knowledge about the empirical domain of one of these theories from knowledge about the domain of the other theory (see [41]). Historically, most examples of epistemic intertheoretic relations have been taken from physics. They include the relation between chemistry and atomic physics, between rigid body mechanics and particle mechanics, and between thermodynamics and statistical mechanics. In the last thirty years, philosophical interest in intertheoretic relations has extended to disciplines like biology (see e.g. [53,58]), ecology (see e.g. [33]), neuroscience (see e.g. [4,55]), and economics (see e.g. [28]). ${ }^{1}$ However, this interest has not been directed at linguistics.

Arguably, the lack of philosophical interest in linguistic intertheory relations cannot be attributed to the absence of linguistic work on intertheory relations or to linguists' disinterest in their subject's methodology. To the contrary: The relations between different linguistic subdisciplines - and between linguistics and neuroscience - have been the topic of much recent research (see e.g. $[10,48])$. The availability of large computerized text corpora and the possibility of statistically probing and manipulating linguistic data sets have further lately effected a boost of interest in linguistic methodology (see the recent textbooks [35], [50], and [47]). The absence of work on linguistic intertheory relations can then only be explained (i) by the fact that philosophers of science are not familiar with theories from linguistics, (ii) by the fact that linguists are not familiar with philosophical models of intertheory relations (assuming that these models have a satisfactory fit with relations between theories in linguistics), or (iii) by the fact that philosophers and/or linguists have little interest in developing or explicating ${ }^{2}$ new models of these relations (assuming that the familiar models do not have a satisfactory fit with relations between theories in linguistics).

In this paper, we explore a particular type of linguistic intertheory relation which does not allow for an analysis through the familiar models of intertheoretic relations from the philosophy of science. This type is instantiated by the relation between linguistic syntax and semantics in a Montague-style formal framework. The combination of these two theories has originally been described as Montague Grammar, or as Montague semantics (after the work of the logician Richard Montague; see [37-39]). Since Montague semantics already specifies - next to the two related theories - the relation between these theories (which aids the formulation of a model of this relation), it is partic-

\footnotetext{
1 The investigated theory-pairs include classical genetics and molecular genetics (or biochemistry), ecology and molecular biology, psychology and computational neuroscience, and macroeconomics and microeconomics.

2 We will see that there, in fact, are models of intertheory relations in linguistics. However, since these models only play a peripheral role in linguistic practice, they are rarely explicitly discussed.
} 
ularly suitable for our purposes. The relative simplicity of this model (w.r.t. later, more sophisticated analyses of the syntax-semantics relation) facilitates the initial presentation and epistemological motivation of this model. Subsequent developments of this model will then capture more intricate aspects of this relation.

The poor fit of the familiar models of intertheoretic relations with the linguistic syntax-semantics relation (see above) is due to the fact that linguistic syntax and semantics describe the behavior of different target domains (i.e. grammatical structures vs. lexical-compositional meanings) and that the familiar models of intertheoretic relations are restricted to theories with the same (or largely overlapping) target domains. Since Montague's syntax-semantics relation obtains between theories with different target domains, this relation adds a new member to the spectrum of intertheoretic relations. We will see that this new relation has a weaker reductive force than the best-studied intertheoretic relation, i.e. Nagelian reduction ${ }^{3}$ [41] (or than its generalized variant, Nagel-Schaffner reduction $[52,53])$, but a stronger reductive force than undirected intertheoretic dependency relations (see e.g. $[13,23,36]$ ). We expect that the development of our model of Montague's syntax-semantics relation will show the importance of investigating intertheory relations in linguistics, that it will help compensate for the absence of models for (one type of) these relations, and that it will yield new insight into the range of different intertheory relations.

We have mentioned above that the reductive force of Montague's syntaxsemantics relation lies between undirected dependency relations and Nagelian reduction. The different types of intertheoretic relations are related by family resemblance. Like Nagelian reduction, Montague's relation serves to derive a proposition of one theory from a proposition of the other theory. As a result, our new type of intertheoretic relation shares the rationale of Nagelian reduction: The relation between linguistic syntax and semantics promotes cognitive economy and simplicity, explains the success of one theory in terms of the success of the other theory, establishes the theories' relative consistency, and effects a mutual flow of confirmation between the two theories. To support the introduction of our new, Montague-inspired, model of intertheoretic relations, the paper will show that this model shares the epistemological advantages of the Nagelian model. ${ }^{4}$

The paper is organized as follows: Section 2 presents a Montague-style framework for the analysis of natural language syntax, semantics, and their relation and contrasts our model of this relation with the model of reduction from Nagel [41]. To prepare the epistemological analysis of our model, Section 3 reviews the relevant concepts from Bayesian confirmation and network theory.

\footnotetext{
3 For many years, Nagelian reduction has been considered a dead end. The present paper rejects this assumption. This stance is motivated by the observation (recorded in [14]) that Schaffner's [53] revised model of Nagelian reduction overcomes the problems of Nagel's original model. For the present purposes, it will suffice to focus only on the Nagelian model. We outline a Schaffner-style extension of our model in Section 5.2.

4 The epistemic advantages of Nagelian reduction are shown in [15].
} 
Section 4 motivates the introduction of our new, Montagovian, model of the syntax-semantics relation. To this aim, it gives a Bayesian analysis of the syntax-semantics relation before and after the establishment of Montague's syntaxsemantics relation and shows that, after the establishment of this relation, the two theories are confirmatory of one another. We close the paper by suggesting a number of extensions to our Montagovian model, which may be developed in future work. These include the generalization of the presented model to pairs of multi-proposition theories (see Sect. 5.1) and its incorporation into a sophisticated variant of Schaffner's [52] revised model of Nagelian reduction (see Sect. 5.2).

Our paper brings together two typically disjoint research areas: linguistic syntax/semantics and Bayesian philosophy of science. We show that the mutual application of these areas yields new insights in both domains. In particular, by investigating the linguistic syntax-semantics relation, philosophers of science will become acquainted with a new, non-canonical example of epistemic intertheoretic relations that resists an analysis through the familiar model(s). By adapting the familiar model of intertheory relations from the philosophy of science (i.e. Nagelian reduction) to Montague's linguistic syntax-semantics relation, linguists will obtain a custom-fit model of this relation which improves upon the fit of the Nagelian model. Bayesian epistemology provides a new tool for evaluating the confirmation of linguistic theories by empirical evidence.

We close this introduction with a disclaimer about the goal of our paper: This paper takes a first step towards developing a model of a new type of intertheoretic relation which is instantiated by Montague's analysis of the syntaxsemantics relation. As a result, the paper develops a simple model of a very small fragment of linguistic syntax and semantics that will require significant further development and extension. In virtue of its simplicity, the developed model will fail to accommodate a fair number of properties of natural language syntax and semantics which are captured by contemporary linguistic theories. In virtue of its domain's small size, the model will only give semantic accounts of some few syntactic phenomena. Since syntax is structurally much richer than semantics, even the intended (i.e. sophisticated, maximal-scope) model will prove unable to explain all syntactic phenomena. ${ }^{5}$ The need to accommodate these phenomena through extra-semantic mechanisms (see Sect. 5.2) witnesses the weak reductive force of the discussed relation (vis-à-vis reduction proper).

\section{Montague's Syntax-Semantics Relation}

To provide the relevant background for our analysis of the syntax-semantics relation, we first present the two linguistic theories (or the two parts ${ }^{6}$ of the the-

\footnotetext{
5 The relevance of word order for the grammaticality of a sentence is a case in point.

6 Since syntax and semantics cover different target domains (see Sections 1 and 2.2), they are often described as parts of the same theory, rather than as two distinct theories. Our choice of these two parts/theories is motivated by the fact that, in addition to formulating a formal theory of syntax and semantics, Montague's framework already provides an analysis
} 
ory) that we aim to relate. ${ }^{7}$ Section 2.1 describes the elements of the two theories and identifies the mechanism which connects these theories. Section 2.2 compares a Montague-style account of the syntax-semantics relation with the model of reduction from Nagel [41]. To prepare the Bayesian analysis of our new type of intertheoretic relation, Section 2.3 identifies Montague's rules for the formation of complex syntactic and semantic structures with the objects of probabilistic evaluations.

\subsection{Montague's 'Two Theories'-Theory}

Montague-style frameworks for the analysis and interpretation of natural language syntax constitute a milestone in the understanding of the linguistic syntax-semantics relation. At the end of the 1960s, there did not exist a sufficiently well-developed formal semantic theory which could be used for the systematic interpretation of natural language. ${ }^{8}$ Montague [38] (see [37, 39]) provides such a theory. ${ }^{9}$ The latter is a model of Church's [11] typed lambda calculus, which contains a designated domain of semantic objects for each syntactic category. Over the last forty-five years, Montague's framework (today called Montague-style formal semantics, or simply Montague semantics) has evolved into the standard theory of compositional linguistic semantics and the syntax-semantics relation. Below, we present a simplified, modern version of Montague's framework that incorporates some subsequent developments. ${ }^{10}$

To enable the systematic interpretation of natural language, Montaguestyle semantics assumes that the semantic objects in the domains of the interpreting model are associated with expressions from distinct syntactic categories $^{11}$ and that the model's rules for the formation of semantic objects are as-

of their relation. This analysis facilitates the work of the philosopher of science, who is responsible for the construction of a model of this relation. Our choice of the syntax-semantics pair is further motivated by the understanding that the ready availability of an analysis of the syntax-semantics relation indicates the linguistic relevance of this (type of) relation.

7 Note that our discussion of the syntax-semantics relation regards syntax and semantics as linguistic theories. As a result, our insights into the syntax-semantics relation cannot be straightforwardly transferred to approaches to formalizing scientific theories (esp. to the syntactic vs. semantic view of scientific theories).

8 Thus, Montague [38] writes, "It is clear [.. .] that [with the exception of [37]] no adequate and comprehensive semantical theory has yet been constructed" (p. 222). Chomsky [9] supports this claim by stating that "In the domain of semantics there are [...] problems of fact and principle that have barely been approached, and there is no reasonably concrete or welldefined 'theory of semantic representation' to which one can refer" (p. 183).

9 At around the same time, similar theories were developed by, a.o., Lewis [34], Cresswell [12], and Bartsch and Vennemann [3].

10 These developments include the interpretation of proper names as individuals (rather than as sets of properties of individuals). This interpretation has been proposed in Flexible Montague Grammar (see [26, 27, 44]).

11 Notably, the designators of semantic objects (which are terms of Montague's logic IL) are not themselves linguistic expressions. In particular, since IL is (Henkin-)complete, the conflation of the language of IL and of natural language would trivialize our reductive endeavor. 
sociated with the familiar syntactic rules. ${ }^{12}$ It identifies categorial grammar [1] (see [40]) as the syntactic theory which best facilitates this correspondence. This theory describes syntax as an algebra over the set of linguistic expressions $\mathcal{E}=\left(\mathcal{E}_{\mathrm{N}} \cup \mathcal{E}_{\mathrm{V}} \cup \mathcal{E}_{\mathrm{S}} \cup \ldots\right)$ which generates complex expressions (e.g. the sentence Bill walks $\in \mathcal{E}_{\mathrm{s}}$ ) from simpler expressions (e.g. from the proper name Bill $\in \mathcal{E}_{\mathrm{N}}$ and the intransitive verb walk $\in \mathcal{E}_{\mathrm{V}}$ ) via syntactic operations like concatenation.

For the combination of proper names with intransitive verbs, the behavior of concatenation is governed by the rule $\mathbb{G}_{\mathrm{S}}$ (see [39, rule $\left.\mathrm{S} 4\right]$ ). In this rule, $\left[A B^{\prime}\right]$ is the result of concatenating the expressions $A$ and $B^{\prime}$ (in that order), where $A$ is a singular name (e.g. Bill) and $B^{\prime}$ is the result (e.g. walks) of replacing the verb $B$ (here, walk) by its third person singular present form.

$\mathbb{G}_{\mathrm{s}}$. If $B \in \mathcal{E}_{\mathrm{v}}$ and $A \in \mathcal{E}_{\mathrm{N}}$, then $\left[A B^{\prime}\right] \in \mathcal{E}_{\mathrm{s}}$.

To facilitate the presentation of Montague-style semantics, we limit ourselves to a syntactically poor fragment of English that only contains proper names, intransitive verbs, and declarative sentences or clauses. As a result, the behavior of concatenation is only governed by the rule $\mathbb{G}_{S}$, such that the set of syntactic rules, $\mathbb{G}$, is identified with the singleton $\left\{\mathbb{G}_{\mathrm{S}}\right\}$. Our fragment is then identified with the closure of the set $\mathcal{E}$ under the rule $\mathbb{G}_{\mathrm{S}}$. By introducing other concatenation rules, we can easily extend our fragment to syntactically more diverse subsets of English.

We next turn to the semantic theory: We have noted above that formal semantic models contain a designated semantic domain for each syntactic category. Thus, a model for our small fragment will include a domain of individuals $\mathcal{D}_{\mathrm{N}}$, a domain of properties of individuals $\mathcal{D}_{\mathrm{v}}$, and a domain of truth-values (or of propositions) $\mathcal{D}_{\mathrm{S}}$. The interpretation function $I$ relates the domains in $\mathcal{E}$ and $\mathcal{D}=\left(\mathcal{D}_{\mathrm{N}} \cup \mathcal{D}_{\mathrm{V}} \cup \mathcal{D}_{\mathrm{S}}\right)$ by assigning, to each $\mathcal{E}_{k}$-expression, $c$, (where $k$ designates a syntactic category) a model-theoretic object, $C$, in the semantic domain $\mathcal{D}_{k}$, such that $I(c)=C$. In this way, the function $I$ will assign, to the name Bill, the individual Bill (i.e. 옷 and will assign, to the verb walk, the property 'walk'.

From the above interpretations, truth-values (here, the truth-value of the sentence Bill walks) are obtained via a semantic correlate, $\mathbb{S}_{\mathrm{S}}$, of the rule $\mathbb{G}_{\mathrm{S}}$ (see [39, rule T4]). In the specification of the rule $\mathbb{S}_{\mathrm{s}}$, we abbreviate ' $I(c)$ ' as ' $\llbracket \rrbracket \rrbracket '$ ' ‘ $\llbracket B \rrbracket(\llbracket A \rrbracket)$ ' is interpreted as the functional application of the denotation of $B$ to the denotation of $A$ :

$\mathbb{S}_{\mathrm{s}}$. If $\llbracket B \rrbracket \in \mathcal{D}_{\mathrm{v}}$ and $\llbracket A \rrbracket \in \mathcal{D}_{\mathrm{N}}$, then $\llbracket B \rrbracket(\llbracket A \rrbracket) \in \mathcal{D}_{\mathrm{S}}$.

As a result of the above, the semantics of our fragment constitutes an algebra $\langle\mathcal{D}, \mathbb{S}\rangle$ over the set of basic model-theoretic objects (where $\mathbb{S}=\left\{\mathbb{S}_{\mathrm{S}}\right\}$ ). The described relation between the syntactic and the semantic algebra is captured in Figure 1.

Our previous presentation has suggested the existence of an injective homomorphism between the syntactic and the semantic algebra. However, in prac-

\footnotetext{
12 This is what Bach [2] has called the 'rule-by-rule' approach to the syntax-semantics correspondence (see [45, p. 13]).
} 


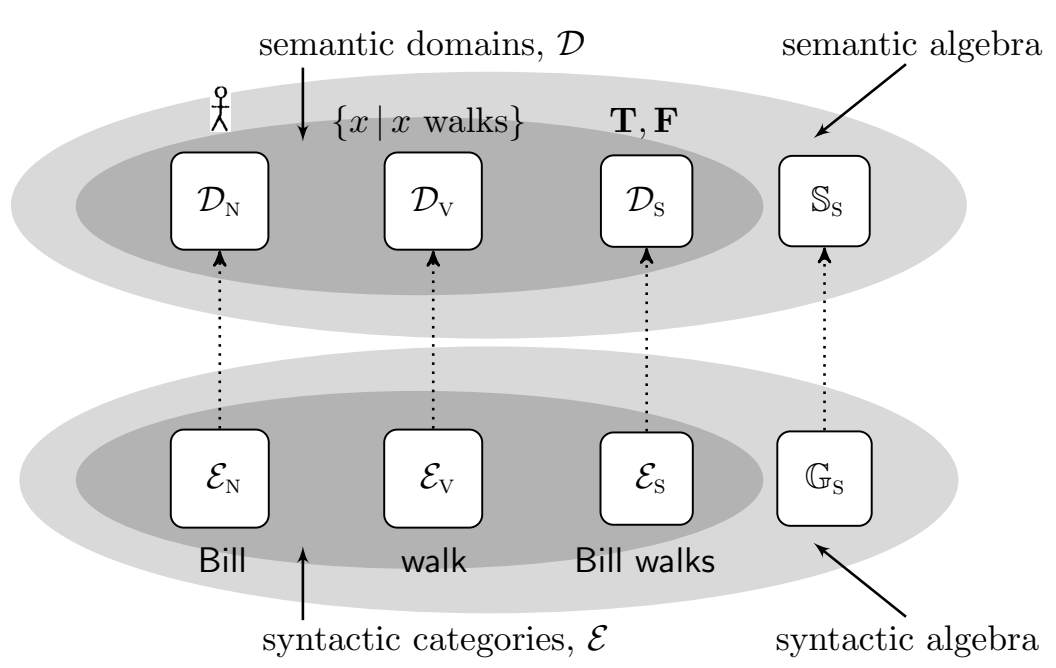

Fig. 1 The (injective) syntax-semantics map.

tice, the situation is more complicated: For example, since Montague interprets intransitive verbs (e.g. walk $\in \mathcal{E}_{\mathrm{V}}$ ) and common nouns (e.g. man $\in \mathcal{E}_{\mathrm{C}}$ ) as elements of the same semantic domain, $\mathcal{D}_{\mathrm{V}},{ }^{13}$ the homomorphism $h$ between syntax and semantics is not injective (see Figure 2).

The situation is further complicated by expressions (e.g. presuppositional verbs like suck) which take complements of different syntactic categories (here: determiner phrases [DPs], including proper names; see (ex.a)) and complement phrases [CPs] (see (ex.b)).

(ex) a. [DPRain] sucks. / [DPBill] sucks.

b. ${ }_{\mathrm{CP}}$ That it is raining today] sucks.

The category-neutrality of these expressions may be taken to suggest that different occurrences of the same expression are interpreted in different semantic domains, such that the syntax-semantics relation is also not a function (see [27,44]). Several modern accounts (e.g. [51, pp. 341-342], [20]) explain the above DP/CP-neutrality by assuming that expressions which take complements of different categories are lexically ambiguous. On these accounts, suck is polysemous between the verb suck ${ }_{1}$ whose occurrences combine with a DP (and are interpreted as elements of the domain $\mathcal{D}_{\mathrm{V}}$ ) and the verb suck ${ }_{2}$ whose occurrences combine with a CP (and are interpreted as elements of the domain of properties of propositions, $\left.\mathcal{D}_{\mathrm{V}^{\prime}}\right)$. The resulting relation between the syntactic and the semantic algebra is captured in Figure 2.

\footnotetext{
13 As a result of this interpretation, distributional differences between common nouns and intransitive verbs can only be explained through syntax. Montague does not regard this delegation of explanatory power as a defect of his framework. To the contrary: He attributes "the fact that Ajdukiewicz's proposals have not previously led to a successful syntax" to "the failure to pursue the possibility of syntactically splitting categories originally conceived in semantic terms" (see [39, p. 249, fn. 4]).
} 


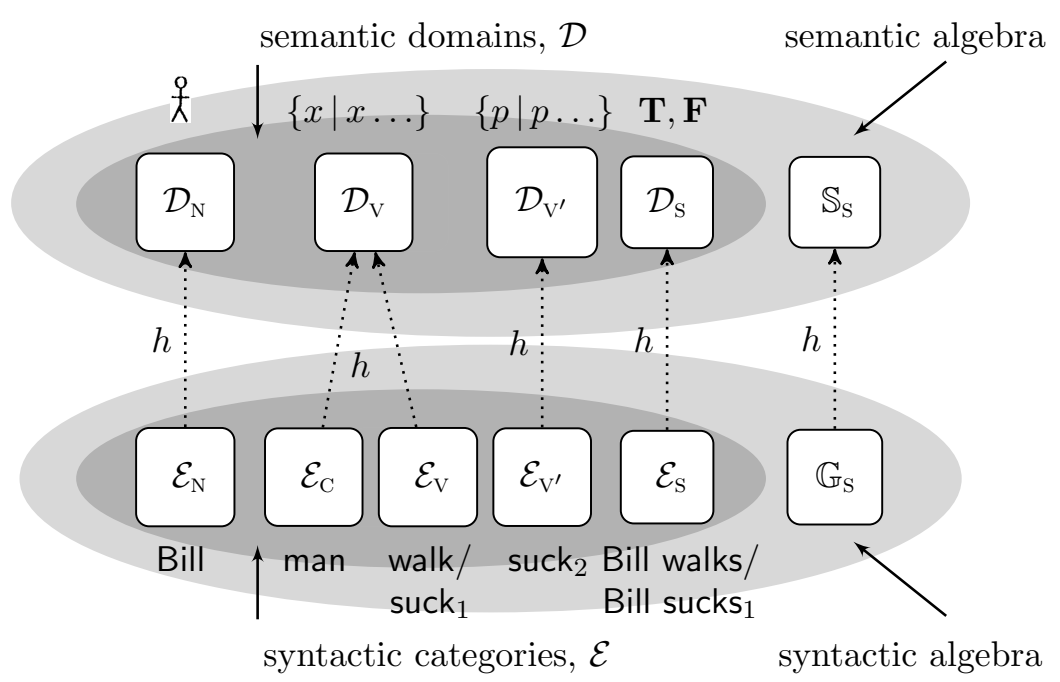

Fig. 2 The non-injective syntax-semantics map.

More recent accounts explain the above DP/CP-neutrality by assuming a covert definite determiner that combines with a $\mathrm{CP}$ in the subject of presuppositional verbs like suck to form a DP (e.g. Kastner [30]; see $[22,56]$ ) or by assuming a nominalization operation at the level of Logical Form that shifts truth-values (or propositions) to individuals, or to generalized quantifiers over individuals (see $[8,49]$ ). On both of these accounts, CP-taking occurrences of verbs like suck are still interpreted in the domain $\mathcal{D}_{\mathrm{V}}$, such that they preserve the injective map from $\mathcal{E}_{\mathrm{V}}$ to $\mathcal{D}_{\mathrm{V}}$. The availability of different (syntactic and semantic) explanations for the DP/CP-neutrality of suck illustrates the intricate connection between syntax and semantics.

Remarkably, the non-injective nature of the Montagovian syntax-semantics relation does not have an effect on the injectivity of the homomorphism between syntactic and semantic rules (at least for the above examples). The reason for this is that the combinatorial behavior of common nouns and their interpretation is governed by different syntactic and semantic rules ${ }^{14}$ than the behavior of intransitive verbs, such that common nouns require the introduction of different syntactic and semantic rules. A similar observation holds for the combinatorial behavior of (the interpretations of) CP-selecting intransitive verbs like suck $_{2}$.

To keep the to-be-developed model as simple as possible, we temporarily ignore these other rules. The extension of the above via multiple pairs of syntactic and semantic rules is discussed in Section 5.1. Since Kastner [30] analyzes

\footnotetext{
14 In particular, common nouns (e.g. man) combine with a determiner (e.g. the/a, interpreted as a function from properties of individuals to sets of such properties) to form a DP (here: the man/a man, interpreted as a set of properties of individuals) (see [39, rules S2, S4; $\mathrm{T} 2, \mathrm{~T} 4])$.
} 
the occurrence of the subject from (ex.b) as a DP, his account handles the formation and interpretation of (ex.b) through the familiar rules $\mathbb{G}_{\mathrm{S}}$ and $\mathbb{S}_{\mathrm{S}}$.

We close our presentation of the Montagovian account of the linguistic syntax-semantics relation with an observation about the aims and scope of linguistic syntax and semantics, and about the different kinds of data which they are designed to capture. In particular, while the aims and scope of categorial grammar are completely standard ${ }^{15}$, the aims and scope of model-theoretic semantics - as presented here - only constitute a proper subclass of the typical aims and scope of semantics. This is due to our focus on semantic rules like $\mathbb{S}_{\mathrm{S}}$, which capture the 'combinability' of semantic interpretations:

Traditionally, linguistic semantics ${ }^{16}$ (incl. Montague-style formal semantics) is concerned with the compositional interpretation of natural language sentences and with the sentences' truth- and entailment-conditions. Relevant semantic data include speakers' ability to 'compute' the compositional meaning of a sentence from the meanings of the sentence's syntactic constituents and their mode of combination, speakers' evaluation of sentential truth or falsity (in a given context), and speakers' judgement about the obtaining of inferential relations between sentences.

Our emphasis on the 'combinatorics' of linguistic meanings (see the rule $\mathbb{S}_{\mathrm{S}}$ ) suggests a focus on semantic understanding, i.e. on the compositional computation of sentence meaning. This computation will succeed for pairs of expressions (e.g. the string [Bill] [walks]) which allow the application of the semantic value (or 'meaning') of one expression to the semantic value of the other expression (here: the application of a property of individuals (i.e. walk) to an individual (i.e. Bill)), and will fail for pairs of expressions (e.g. the string [Bill] [every woman]) which do not allow this application.

Remarkably, semantic understanding (or semantic understandability) of a given sentence is strongly reflected in the sentence's grammaticality. This is due to the 'type-checking' role of syntax, which rejects expressions (e.g. the ungrammatical string [Bill] [every woman] from above) whose constituent expressions cannot be meaningfully combined. ${ }^{17}$ The non-combinability of these constituents then explains the lack of meaningfulness of the larger expression.

As a consequence of our focus on semantic rules, we hereafter largely neglect sentential truth and entailment in favor of grammaticality. This neglect is further justified by the fact that, without specifying the meaning of individual words (or phrases), we cannot evaluate the truth or falsity of sentences con-

\footnotetext{
15 Thus, this theory is concerned with the formation of complex linguistic expressions (e.g. sentences, clauses) from simpler expressions (e.g. individual words, phrases), and is supported by strings of expressions which reflect the assumed process for the formation of complex expressions.

16 We here understand 'semantics' as 'compositional semantics'.

17 Admittedly, the type-checking role of syntax may already involve much of the (presumed) work of compositional semantics. This is illustrated by Kastner's syntactic analysis of (ex.b) as (ex.c), whose subject DP (which is required for a successful type-check of the sentence) is obtained by applying the covert definite determiner $\Delta$ to a CP:
}

(ex) c. $[\mathrm{DP} \Delta[\mathrm{CP}$ That it is raining today $]]$ sucks. 
taining these words (or phrases). Without identifying the rules of Montague's logic IL or the meaning postulates which constrain the set of semantic models, we cannot judge whether one sentence entails another.

Because of our focus on syntactic and semantic rules, the predominant support of semantic rules by semantic understandability, and the reflection of understandability in grammaticality, all salient evidence for the rules in $\mathbb{G}$ and $\mathbb{S}$ lies in strings of expressions whose structure reflects the assumed formation process. As a result, only syntactic rules are supported by direct evidence (s.t. syntax fits the phenomena). Semantic rules are mostly supported by indirect evidence (s.t. the relevant part of the empirical access of semantics is through syntax). The different degrees of directness of support for syntactic and semantic rules motivates our identification of syntax with the phenomenological ${ }^{18}$ (i.e. 'reduced') theory and of semantics with the fundamental (i.e. 'reducing') theory.

The identification of semantics with the fundamental theory is in line with Montague's (historical) view of the primacy of semantics, which regards syntax as a mere "preliminary to semantics" [38, p. 223, fn. 2]. Admittedly, in light of the greater structural richness of syntax w.r.t. semantics, the identification of semantics with the fundamental theory has since been much contested (see e.g. $[9,30,56])$. However, to reflect the Montagovian idea behind the presented model of the syntax-semantics relation - and to capture the direct support of rules in $\mathbb{G}$-, this paper will nevertheless identify semantics with the fundamental theory. The adequacy of the syntax-semantics relation which is obtained on this model will determine the relative success of the proposed model. Proponents of the 'fundamentality' of syntax may use a close version of the presented model (with $r$ replaced by $h$ as the relevant inter-rule relation) to capture the relation between semantics and syntax.

This completes our presentation of a Montague-style account of the syntaxsemantics relation. We next compare this account to the model of intertheoretic reduction from [41].

\subsection{Montague's Syntax-Semantics Relation and Intertheoretic Reduction}

In Section 1, we have described Montague's syntax-semantics relation as the instantiation of a specific type of intertheoretic relation. To emphasize the similarities and differences of our Montagovian model to the account of reduction from Nagel [41, Ch.11], we next describe the relation between syntax and se-

\footnotetext{
18 Our use of the terms phenomenological and fundamental is in accordance with the treatment of theories in physics. Our adoption of these terms in linguistics is motivated by the wish to avoid reference to the 'reduced' and the 'reducing' theory (see Sect. 1). Nothing more depends on this terminology.
} 
mantics on the Nagelian model. ${ }^{19}$ We then compare the Montagovian account of intertheoretic relations to the Nagelian account.

In the following, we assume a phenomenological theory, $\mathcal{T}_{2}$, and a fundamental theory, $\mathcal{T}_{1}$. These theories are associated with sets of propositions $T_{2}=\left\{T_{2}^{1}, T_{2}^{2}, \ldots\right\}$ and $T_{1}=\left\{T_{1}^{1}, T_{1}^{2}, \ldots\right\}$, respectively. As is well known, Nagelian reduction is a three-step process that involves the connection of terms in the non-logical vocabulary of $T_{1}$ and $T_{2}$ via bridge laws (step (i)), the substitution of the terms from $T_{1}$ by their bridge-law correspondents from $T_{2}$ (step (ii)), and the derivation (via auxiliary assumptions) of every proposition in $T_{2}$ from the result of replacing the relevant terms in a proposition of $T_{1}$ by their bridge-law correspondents (step (iii)) (see [41, pp. 353-354]).

For example, on the Nagelian account, the reduction of the theory of the ideal gas from thermodynamics [TD] to the kinetic theory of gases from statistical mechanics $[\mathrm{SM}]$ involves (i) the connection of the temperature variable $T$ from TD with the term for the mean kinetic energy of a particle $\left\langle E_{\text {kin }}\right\rangle$ from SM, (ii) the substitution of $\left\langle E_{\text {kin }}\right\rangle$ by $T$ in the laws of the theory of the ideal gas, and (iii) a demonstration of the fact that the result of this substitution follows from the Newtonian equation of motion and the definitions of pressure and kinetic energy (assuming that the molecules are point particles, that they collide elastically, and that the velocity distribution is isotropic). Because of the general familiarity of the Nagelian model of reduction - and since the above sketch of this model contains all relevant information for an insightful comparison with our Montagovian model -, we refrain from describing the Nagelian model in greater detail. For a careful presentation of (a modern version of) this model, the interested reader is referred to [14].

Analogously to the above, the Nagelian reduction of the linguistic syntaxsemantics pair would involve (i) the connection of the names of the elements in $\mathcal{D}$ and $\mathcal{E}$ with the designators of the semantic and syntactic operations from the rules in $\mathbb{S}$ and $\mathbb{G}$, (ii) the substitution of the names of the elements in $\mathcal{D}$ and the operations from the rules in $\mathbb{S}$ by their bridge-law correspondents from $\mathcal{E}$ and $\mathbb{G}$ in the designators of the rules from $\mathbb{S}$, and (iii) the derivation of every rule (or 'proposition') in $\mathbb{G}$ from the corresponding proposition in $\mathbb{S}$.

In particular, step (i) connects $\mathcal{E}_{\mathrm{N}}$ with $\mathcal{D}_{\mathrm{N}}, \mathcal{E}_{\mathrm{C}}$ and $\mathcal{E}_{\mathrm{V}}$ with $\mathcal{D}_{\mathrm{V}}, \mathcal{E}_{\mathrm{V}^{\prime}}$ with $\mathcal{D}_{\mathrm{V}^{\prime}}$, and $\mathcal{E}_{\mathrm{S}}$ with $\mathcal{D}_{\mathrm{S}}$, and connects expression concatenation, $\{\cdot\}$ with function application, $\lambda y \lambda x . y(x) .{ }^{20}$ Step (ii) converts a copy of the rule $\mathbb{S}_{\mathrm{S}}$ (in (1a)) into the rule from $(1 \mathrm{~b})$ by replacing every occurrence of ' $\mathcal{D}_{\mathrm{N}}$ ' by ' $\mathcal{E}_{\mathrm{N}}$ ', of ' $\mathcal{D}_{\mathrm{V}}$ ' by ' $\mathcal{E}_{\mathrm{V}}$ ', and of ' $\mathcal{D}_{\mathrm{S}}$ ' by ' $\mathcal{E}_{\mathrm{S}}$ ', and by replacing every occurrence of ' $\lambda y \lambda x . y(x)$ ' by the operator ' $\{\cdot\}$ '. Step (iii) trivially derives a variant of the rule $\mathbb{G}_{\mathrm{S}}$ from the result of this conversion.

\footnotetext{
19 We will see below that - because of the interpretation of bridge laws as statements about the inclusion relation between the extensions of terms of the two theories - the Nagelian model can, in fact, not be applied to the syntax-semantics pair. To allow a direct comparison of the Montagovian with the Nagelian model, we temporarily ignore this inapplicability.

20 To accommodate the word-order profiles of different languages (e.g. 'subject-verb-object' vs. 'verb-subject-object'), we here use an order-invariant version, $\{\cdot\}$, of the concatenation operation [ . ] from $\mathbb{G}_{\mathrm{S}}$. The order-(in)variance of concatenation is discussed below and in Section 5.2 .
} 
(1) a. If $Y \in \mathcal{D}_{\mathrm{V}}$ and $X \in \mathcal{D}_{\mathrm{N}}$, then $Y(X) \in \mathcal{D}_{\mathrm{S}}$.

b. If $Y \in \mathcal{E}_{\mathrm{V}}$ and $X \in \mathcal{E}_{\mathrm{N}}$, then $\{Y X\} \in \mathcal{E}_{\mathrm{S}}$.

c. If $Y \in \mathcal{E}_{\mathrm{C}}$ and $X \in \mathcal{E}_{\mathrm{N}}$, then $\{Y X\} \in \mathcal{E}_{\mathrm{S}}$.

Note that, since the rule (1c) is not a syntactic rule of English (i.e. it yields ill-formed strings of the form [Bill] [man]), we neglect the result, (1c), of replacing ' $\mathcal{D}_{\mathrm{V}}$ ' by ' $\mathcal{E}_{\mathrm{C}}$ ' in $\mathbb{S}_{\mathrm{S}}$. The possibility of using the Nagelian model to derive syntactic rules which are not members of $\mathbb{G}$ again illustrates that Montague's syntax-semantics relation has a weaker reductive force than Nagelian reduction. We will see below that our model of Montague's syntax-semantics relation avoids the obtaining of non- $\mathbb{G}$ rules like (1c).

The above example shows that our Montague-style model of the syntaxsemantics relation and the Nagelian model of intertheoretic reduction agree with respect to the connectability of the two theories (cf. step (i)). Yet, while Nagel's model satisfies this requirement through the formulation of syntactic bridge laws (which connect pairs of terms in the vocabulary of the two theories), our Montagovian model satisfies this requirement through the assumption of a relation $r:=h^{-1}$ between the objects of the semantic and the syntactic algebras ([38], [45, pp. 15-20]; see Figure 3). This relation generalizes the inverse image of the interpretation function $I$ from Section 2.1, such that $r\left(\mathcal{D}_{k}\right)=\left\{I^{-1}(a) \mid a \in \mathcal{D}_{k}\right\} \subseteq \mathcal{E}_{k}$. Since the relation $r$ also establishes connections between the rules of the two theories (s.t. $r\left(\mathbb{S}_{\mathrm{S}}\right)=\mathbb{G}_{\mathrm{S}}$ ), our Montagovian model obviates Nagel's substitution step (ii) and, thus, avoids the obtaining of non- $\mathbb{G}$ rules like (1c).

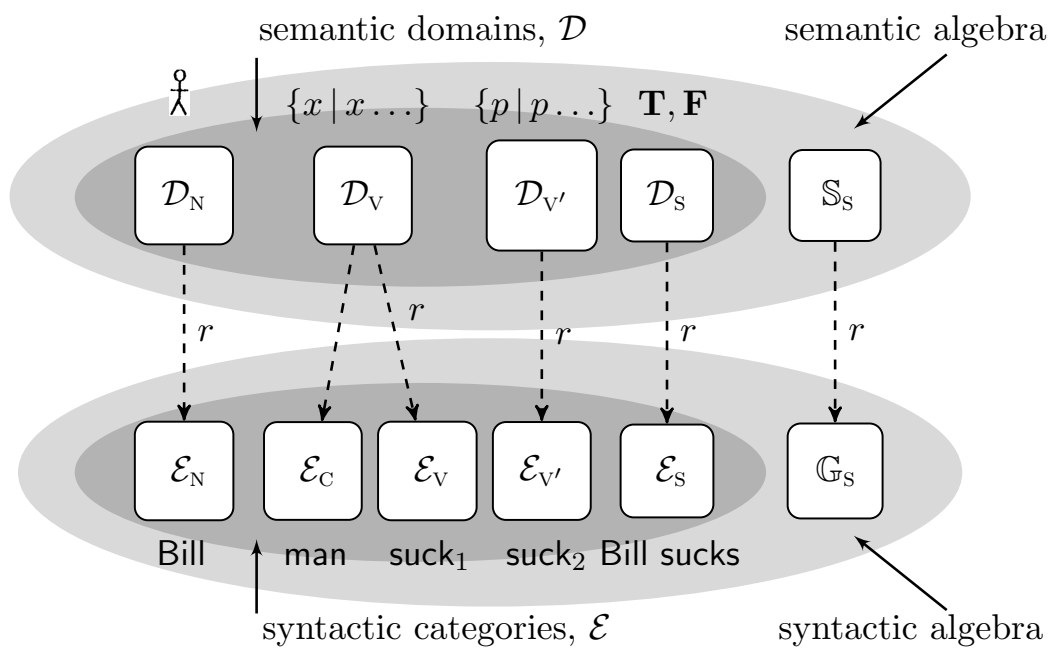

Fig. 3 The semantics-syntax relation.

In our model of Montague's syntax-semantics relation, the replacement of Nagelian bridge laws by the relation $r$ is made necessary by the definition of 
bridge laws as inclusion relations between the extensions of terms in $T_{1}$ and $T_{2} \cdot{ }^{21}$ As a result of this definition, it holds for all pairs of terms, $t_{1}$ and $t_{2}$, from $T_{1}$ and $T_{2}$, that $t_{2}$ applies to all objects to which $t_{1}$ applies. Since modeltheoretic semantics and categorial grammar have non-overlapping target domains (s.t. they do not satisfy the inclusion-of-extensions requirement on terms), their objects need to be connected in some other way. The assumption of the relation $r:=h^{-1}$ between the algebraic formulations of $T_{1}$ and $T_{2}$ serves this purpose.

In our Montagovian model, the replacement of bridge laws by the relation $r$ also obviates the specification of the relation between magnitude parameters, which is required in the Nagelian model (see [53]): To accommodate the magnitudes of physical properties (e.g. the degrees Kelvin of a gas' temperature), Nagelian bridge laws specify a functional dependence relation $f$ between the magnitudes, $\tau_{1}$ and $\tau_{2}$, of the properties denoted by the terms $t_{1}$ and $t_{2}$ (s.t. $\left.\tau_{2}=f\left(\tau_{1}\right)\right)$. Since the relevant properties in linguistic syntax and semantics are magnitude-free, our Montagovian model does not (need to) specify such a relation.

Notably, the semantic characterization of connectability, which is adopted in our Montagovian model, also improves upon the adequacy of the Nagelian model - especially upon its proximity to scientific practice. Nagel's model of reduction is based on the syntactic view of scientific theories (see [6, 25]), which reconstructs theories as axiomatic logical systems whose language is bifurcated into observational terms (which refer to directly observable entities) and nonobservational theoretical terms (whose meaning is determined by the axioms of the theory). Their centrality to scientific theories also gives theoretical terms a prominent role in Nagel's model of reduction. However, when establishing relations between theories, scientists do not - above all - link the theories' nonobservational vocabularies (e.g. the thermodynamic term temperature and the statistical mechanical equation ' $\frac{2 n}{3 k}\left\langle E_{\text {kin }}\right\rangle$ '). Rather, they establish relations between the corresponding semantic objects (here: between the concept 'temperature' and the mean kinetic energy of a particle). Our reconstruction of Montague's model of the syntax-semantics relation captures this semantic perspective.

The semantic characterization of connectability is not the only salient property of our model of Montague's syntax-semantics relation: This model is also defined by the non-functional character of the relation $r$ (and by the resulting non-symmetry of the associated intertheoretic relation): The Nagelian and the Montagovian model both characterize reduction as a directed dependency relation. However, the common conception of Nagelian bridge laws as biconditional statements (see [53, p.622], [31, p. 310]) conceals this property. ${ }^{22}$ As a

\footnotetext{
21 This characterization of Nagelian bridge laws is due to Schaffner [53, pp. 614-615] (see [54, pp.411-477]). However, since it generalizes Nagel's categorization of bridge laws as meaning-entailments, conventional stipulations, or matters of fact (see [41, pp. 354-355]), we here treat it as a proper part of Nagel's model.

22 Notably, Nagel himself only demands that bridge laws take the form of one-directional conditional statements (see [41, pp. 354]). The common conception of Nagelian bridge laws
} 
result, this conception represents the Nagelian reduction relation as a symmetric relation. In contrast, the Montagovian model represents this relation as an asymmetric relation. To emphasize the symmetric character of Nagelian reduction (as commonly conceived), we will sometimes describe this relation as an undirected relation. ${ }^{23}$ For simplicity, we hereafter identify Nagel(ian) Reduction with the common conception of Nagelian reduction.

Figure 4 compares Montague's account of the syntax-semantics relation (right) with the Nagelian account of reduction (left).

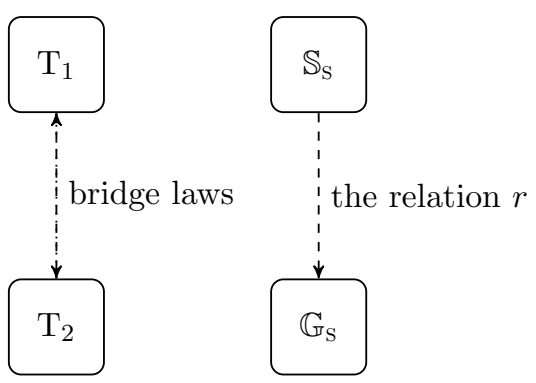

Fig. 4 The Nagelian model (left) and the Montagovian model of intertheoretic relations (right).

Note our use of dashed (rather than dotted) arrows in the above figure (and in Figure 3). This notation is required by the directedness of the syntaxsemantics relation, such that the arrows from Figures 2 and 4 have a different denotation: While the arrows from Figure 2 represent Montague's homomorphism $h$, the arrows in Figure 3 and in the right part of Figure 4 represent its inverse image (i.e. the relation $r$ ).

For future reference, we define intertheoretic relations on the Nagelian and the Montagovian model in terms of their salient properties as follows:

Definition 1 (Nagel Reduction) A type of undirected (i.e. symmetrically represented) dependency relation, described in [41] (see [53]) which is defined by the existence of intertheoretical connections between terms in the nonlogical vocabulary of the two related theories and by the derivability of every proposition in the phenomenological theory from a corresponding proposition in the fundamental theory.

Definition 2 (Montague's Relation) A type of directed (or non-symmetrically represented) dependency relation, inspired by [38], which is defined by the existence of intertheoretical connections between objects of the two related theories and by the resulting trivial derivability of every proposition in the

as biconditional statements can be attributed to the fact that all examples of bridge laws from [41, Ch. 11] are biconditionals.

23 For a discussion of this issue - and for a Nagelian solution -, the reader is referred to [32] and $[14]$. 
phenomenological theory from a corresponding proposition in the fundamental theory.

The commonalities and differences between intertheoretic relations on the Nagelian and the Montagovian model are captured in Figure 5.

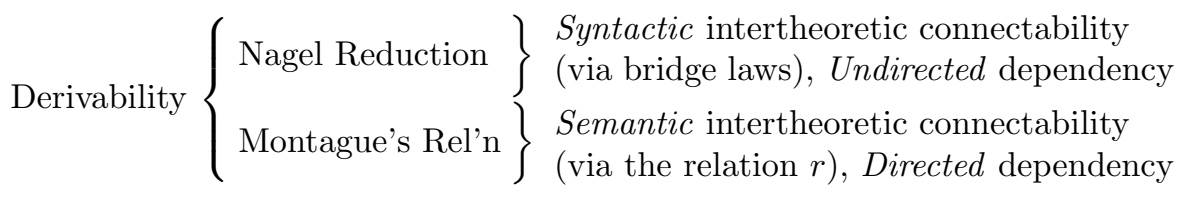

Fig. 5 Intertheoretic relations on the Montagovian vis-à-vis the Nagelian model.

It is clear from the above that our model of Montague's syntax-semantics relation instantiates only one particular type of intertheoretic relation. There are many others, ranging from 'strict' Nagelian Reduction (see Def. 1) via the 'weaker' Nagel-Schaffner reduction $[52,53]$ (see Sect. 5.2) to undirected dependency relations (see e.g. $[13,23,36]$ ). We expect that the relation between model-theoretic semantics and categorial grammar be found in the mid-range of this spectrum.

We close the present subsection with a number of caveats about the linguistic syntax-semantics relation: Our previous considerations have identified Montague's syntax-semantics relation as a weak, i.e. directed, variant of Nagel Reduction. Significantly, however, Montague's syntax-semantics relation is even weaker than has been previously established. This is due to the greater structural richness of syntax in comparison to semantics, such that Montague's syntactic rule $\mathbb{G}_{\mathrm{S}}$ contains more information than the semantic rule $\mathbb{S}_{\mathrm{S}} \cdot{ }^{24}$ Word order and agreement are a case in point: To ensure the 'right' formation of simple English sentences (in which the third person singular form of an intransitive verb combines with a proper name which occurs on its left), Montague's rule $\mathbb{G}_{\mathrm{S}}$ uses the order-sensitive concatenation operation [ · ]. Without this orderspecification, nothing would prevent the concatenation of expressions which violate the language's word-order profile (e.g. subject-verb-object for English). As a result, the rule from (1b) could yield either the expression Walks Bill or Bill walks. This observation contributes to our description of Montague's syntaxsemantics relation as a distinct type of intertheoretic relation, rather than as strong Nagelian reduction.

Our characterization of the linguistic syntax-semantics relation as a weak intertheoretic relation requires one further clarification: All popular accounts of intertheoretic relations (incl. [41]) assume that the phenomenological and the fundamental theory have the same (or largely overlapping) target domains. On this account, the two theories both make more-or-less the same claims (e.g.

\footnotetext{
24 In virtue of this observation, Montague's syntax-semantics relation is also not an instance of the interpretability relation between theories (see [57]). This relation holds of two theories, $T_{1}$ and $T_{2}$, if there exists some extension, $T_{1}^{*}$, of $T_{1}$ via a recursive set of explicit definitions such that every consequence of $T_{2}$ is a consequence of $T_{1}^{*}$ (see $[17$, p. 495]).
} 
about the behavior of a given physical system). We have argued above that this is not the case for our syntax-semantics pair: ${ }^{25}$ While categorial grammar accounts for the well- (or ill-)formedness of syntactic structures, model-theoretic semantics accounts for the compositional properties of these structures' interpretations. Admittedly, the interpretation relation $I$ establishes a firm connection between the objects of the two theories. However, this does not change the fact that the reductive achievement of Montague's syntax-semantics relation will be comparatively weaker than the achievements of (strong or weak) reductions between shared-domain theories.

The admonitions from the last two paragraphs all characterize our new type of intertheoretic relation. While some of these admonitions will be ignored in the rest of this paper, their neglect would distort our representation of the syntax-semantics relation. To enable a Bayesian analysis of our model of this relation, we next discuss the use of probabilities in linguistic syntax and semantics. This discussion is followed by a primer on Bayesian confirmation and network theory (in Section 3).

\subsection{Montagovian Rules and Probabilities}

Our presentation of Montague's theory of the syntax-semantics relation has presupposed the existence of two sets of rules, $\mathbb{G}$ and $\mathbb{S}$, for the formation of complex syntactic and semantic objects. Like hypotheses of any scientific theory, these rules are obtained via the scientific method (discussed, here, for the formulation of the rule $\mathbb{G}_{\mathrm{S}}$ ): Following the isolation of syntactically simple sentences in a given data-set (typically, an electronic text collection like the British National Corpus), linguists abstract information about the sentences' structural properties and propose a hypothesis (here, $\mathbb{G}_{\mathrm{S}}$ ) about their formation. Hypotheses are tested through the analysis of strings of expressions in other (new) corpora: A given string (e.g. the sentence Bill walks) is taken to support the hypothesis if its structure does, and to question the hypothesis if its structure does not reflect the assumed formation process (i.e. if it positively resp. negatively instantiates $\mathbb{G}_{\mathrm{S}}$ ).

To enable a Bayesian analysis of our model of the syntax-semantics relation, we assign a probability to every syntactic and semantic rule. A rule's probability is informed by the frequentist data which are available at the time. Thus, the probability of the truth of the hypothesized rule $\mathbb{G}_{\mathrm{S}}$ will be very high (or low) if a very large (resp. small) percentage of the expressions of the described form instantiates $\mathbb{G}_{\mathrm{S}}$. We expect that the frequentist probability of a given rule will influence a linguist's psychological confidence in the rule's descriptive adequacy. In particular, if a very large (or small) percentage of the

\footnotetext{
25 Notably, relations between theories with different target domains may not be identified with heterogeneous reductions, whose constituent theories do not share the relevant predicates: Many heterogenous reductions (e.g. the reduction of thermodynamics to statistical mechanics) still have a common target domain (here: observable phenomena in macroscopic systems like gases).
} 
expressions of a given form instantiates $\mathbb{G}_{S}$, the linguist's belief in the truth of $\mathbb{G}_{\mathrm{S}}$ will be similarly high (resp. low).

Our previous considerations have defined the probability of a given rule via the frequency of the rule's positive instantiations in a given sample. We have already argued in Section 2.2 that the relation of direct instantiation by linguistic objects is restricted to syntactic rules. We will see below that this observation plays an important role in the confirmation of the two theories before their relation has been established (i.e. in the two theories' pre-'reductive ${ }^{26}$ confirmation) (see Prop. 1, Sect.4.1). The semantic rule $\mathbb{S}_{\mathrm{S}}$ derives its support from the linguistic support of the rule $\mathbb{G}_{\mathrm{S}}$ via the assumption of the relation $r$. The probability of $\mathbb{S}_{\mathrm{S}}$ is thus obtained via the probability of its syntactic counterpart.

This concludes our discussion of the reductive and probabilistic aspects of the Montagovian framework. We precede our introduction to Bayesianism with one final caveat: Importantly, our attribution of probabilities to Montagovian rules does not constitute a probabilistic extension of Montague semantics. The central aim of this paper is methodological, not substantive. Consequently, we do not intend any revisions or additions to (our fragment of) Montague-style formal semantics. The attribution of probabilities is only a means to an end, i.e. the possibility of providing a Bayesian analysis of the presented model of the linguistic syntax-semantics relation. To achieve this end, it will suffice to restrict ourselves to the use of probabilistic variables. While nothing prevents us from inserting actual values, the use of such values is not necessary for the success of our analysis.

\section{A Primer on Bayesianism}

We analyze a rule's evidential support via Bayesian confirmation theory: The central idea of this theory is the interpretation of confirmation as probabilityraising and the associated distinction between two notions of probability, relative to the receipt of a new piece of evidence: The initial, or prior, probability of a proposition $\mathrm{H}$ (for 'hypothesis') is the probability of $\mathrm{H}$ before the evidence $\mathrm{E}$ has been considered. The final, or posterior, probability of $\mathrm{H}$ is the probability after $\mathrm{E}$ has been considered.

Bayesian conditionalization on E requires an update of the prior probability, $\boldsymbol{P}(\mathrm{H})$, to the posterior probability, $\boldsymbol{P}^{\prime}(\mathrm{H})$, of $\mathrm{H}$, where $\boldsymbol{P}^{\prime}(\mathrm{H})$ is typically expressed in terms of the original probability measure, i.e. $\boldsymbol{P}^{\prime}(\mathrm{H})=\boldsymbol{P}(\mathrm{H} \mid \mathrm{E})$, provided that $\boldsymbol{P}(\mathrm{E})>0$. Our use of Bayes' Theorem, a result from probability

\footnotetext{
26 To emphasize the weak nature of this relation w.r.t. reduction proper, we hereafter write reductive in scare quotes.
} 
theory, yields the following expression for the posterior probability of $\mathrm{H}$ :

$$
\begin{aligned}
\boldsymbol{P}(\mathrm{H} \mid \mathrm{E}) & =\frac{\boldsymbol{P}(\mathrm{E} \mid \mathrm{H}) \boldsymbol{P}(\mathrm{H})}{\boldsymbol{P}(\mathrm{E})}=\frac{\boldsymbol{P}(\mathrm{E} \mid \mathrm{H}) \boldsymbol{P}(\mathrm{H})}{\boldsymbol{P}(\mathrm{E} \mid \mathrm{H}) \boldsymbol{P}(\mathrm{H})+\boldsymbol{P}(\mathrm{E} \mid \neg \mathrm{H}) \boldsymbol{P}(\neg \mathrm{H})} \\
& =\frac{\boldsymbol{P}(\mathrm{H})}{\boldsymbol{P}(\mathrm{H})+\boldsymbol{P}(\neg \mathrm{H}) x}
\end{aligned}
$$

In the above, the expression $x:=\boldsymbol{P}(\mathrm{E} \mid \neg H) / \boldsymbol{P}(\mathrm{E} \mid H)$ is the likelihood ratio.

According to Bayesian confirmation theory, a piece of evidence $\mathrm{E}$ confirms the hypothesis $\mathrm{H}$ if the posterior probability of $\mathrm{H}$ (given $\mathrm{E}$ ) is greater than the prior probability of $\mathrm{H}$, i.e. if $\boldsymbol{P}(\mathrm{H} \mid \mathrm{E})>\boldsymbol{P}(\mathrm{H})$. The piece of evidence, E, disconfirms $\mathrm{H}$ if $\boldsymbol{P}(\mathrm{H} \mid \mathrm{E})<\boldsymbol{P}(\mathrm{H})$ and is irrelevant for $\mathrm{H}$ if $\boldsymbol{P}(\mathrm{H} \mid \mathrm{E})=\boldsymbol{P}(\mathrm{H}){ }^{27}$

While the case of two propositions is easy to compute, the confirmatory situation is often much more complicated. This is due to the fact that the respective hypothesis may have a fine structure and that different pieces of evidence may stand in certain probabilistic relations to one another. As we will see in due course, the relation between linguistic syntax and semantics, upon which we focus in this paper, exhibits a similarly high degree of complexity.

Bayesian networks prove to be a highly efficient tool for the computation of the above-described scenarios. ${ }^{28}$ A Bayesian network is a directed acyclical graph whose nodes represent propositional variables and whose arrows encode the conditional independence relations that hold between the variables. In the rest of this paper, we call parent nodes nodes with outgoing arrows and call child nodes nodes with incoming arrows. Root nodes are unparented nodes; descendant nodes are child nodes or the child of a child node, etc.

By the special choice of graph, paths of arrows may not lead back to themselves (s.t. the graph is acyclical). Variables at each node can take different numerical values, which are assigned by the probability function $\boldsymbol{P}$. As a result, Bayesian networks do not only provide a direct visualization of the probabilistic dependency relations between variables, but come along with a set of efficient algorithms for the computation of whichever conditional or unconditional probability over a (sub-)set of the variables involved we are interested in.

We illustrate the use of Bayesian networks by framing the confirmatory relation between the hypothesis $\mathrm{H}$ and a piece of evidence $\mathrm{E}$. To do so, we first introduce two binary propositional variables, $H$ and $E$ (printed in italic script). Each of these variables has two values (printed in roman script): $\mathrm{H}$ or $\neg \mathrm{H}$ (i.e. 'the hypothesized rule is true' resp. 'false'), and $\mathrm{E}$ or $\neg \mathrm{E}$ ('the evidence obtains' resp. 'does not obtain'). The relation between $E$ and $H$ is represented in the graph in Figure 6.

\footnotetext{
27 Bayesianism is presented and critically discussed in [16] and [29]. These texts also discuss Jeffrey conditionalization, which is an alternative updating rule. For an introduction to Bayesian epistemology, the reader is referred to [21] and [24].

28 For an introduction to Bayesian networks, see [43,46]. The monograph [5] discusses applications from epistemology and the philosophy of science and provides a short introduction to the theory of Bayesian networks.
} 


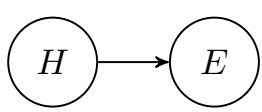

Fig. 6 Bayesian network representation of the dependence between $E$ and $H$.

The arrow from $H$ to $E$ denotes a direct influence of the variable in the parent node on the variable in the child node. The truth or falsity of the hypothesis affects the probability of the obtaining of $E$.

To turn the graph from Figure 6 into a Bayesian network, we further require the marginal probability distribution for each variable in a root node (i.e. the prior probability, $\boldsymbol{P}(\mathrm{H})$, of $\mathrm{H}$ ) and the conditional probability distribution for every variable in a child node, given its parents. In the present case, the latter involves fixing the likelihoods $\boldsymbol{P}(\mathrm{E} \mid \mathrm{H})$ and $\boldsymbol{P}(\mathrm{E} \mid \neg \mathrm{H})$. From these distributions, we can then obtain all other probabilities via Bayesian networks. As will be relevant below, the graph's probability distribution respects the Parental Markov Condition (PMC). According to this condition, a variable represented by a node in a Bayesian network is independent of all variables represented by its non-descendant nodes in the Bayesian network and is conditional on all variables represented by its parent nodes.

\section{Montague's Syntax-Semantics Relation and Confirmation}

Our previous efforts have restricted themselves to the presentation of our Montagovian model of the linguistic syntax-semantics relation. To motivate the introduction of this model as a model of a new type of intertheoretic relation (in addition to the established Nagelian model), we next provide a Bayesian analysis of this model. This analysis will be used to show that the proposed model shares the epistemological advantages of the Nagelian model.

To simplify the Bayesian analysis of our model of Montague's syntaxsemantics relation, and to facilitate its comparison with the Bayesian analysis of Nagel-Schaffner reduction from [15], we focus on the relation between the rules $\mathbb{G}_{\mathrm{S}}$ and $\mathbb{S}_{\mathrm{S}}$. These rules are associated with the propositional variables $G$, respectively $S{ }^{29}$ The intertheoretic relation between linguistic syntax and semantics can then be represented via the graph in Figure 8. For simplicity, we assume that the rule $\mathbb{G}_{\mathrm{S}}$ is supported by exactly one (set of) piece(s) of evidence. As has been explained in Section 2.3, we take evidence for a given syntactic rule to be an intuitively well-formed linguistic expression whose structure reflects the rule's assumed formation process. The replacement of the arrows from Figures 1 to 4 by arrows of the form $\longrightarrow$ (see Fig. 6) is motivated by our interest in probabilistic dependence relations between propositional variables (rather than in the relation $r$ ). Below, these arrows capture the dependence of the probability of the truth of the syntactic rule $\mathbb{G}_{\mathrm{S}}$ on the

\footnotetext{
29 The restriction to singleton sets of rules (s.t. $\mathbb{G}=\left\{\mathbb{G}_{\mathrm{S}}\right\}$ and $\mathbb{S}=\left\{\mathbb{S}_{\mathrm{S}}\right\}$ ) enables us to drop the subscript 's' from $G_{\mathrm{S}}$ and $S_{\mathrm{S}}$.
} 
probability of the truth of the semantic rule $\mathbb{S}_{\mathrm{s}}$. The conditional dependency of $\mathbb{G}_{\mathrm{S}}$ on $\mathbb{S}_{\mathrm{S}}$ enables us to obtain an aligned chain of arrows. As a result, we can represent a flow of evidence from the syntactic to the semantic theory.

Figures 7 and 8 display the graphs associated with the dependence relations between $S, G$, and $E$ before and after the establishment of Montague's syntaxsemantics relation.

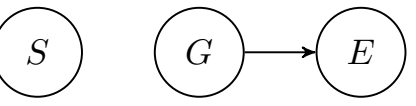

Fig. 7 Pre-'reductive' dependence relations between $S, G$, and $E$.

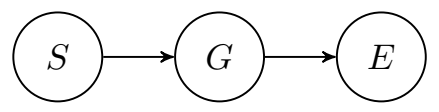

Fig. 8 Post-'reductive' dependence relations between $S, G$, and $E$.

We determine the confirmation of $\mathrm{S}$ and $\mathrm{G}$ via their relevant probabilities, beginning with the pre-'reductive' situation (in Sect. 4.1; see Fig. 7). The comparison of this situation with the post-'reductive' situation (in Sect.4.3; see Sect. 4.2, Fig. 8) will show that Montague's relation between linguistic syntax and semantics raises the joint (prior and posterior) probabilities of the two theories and improves the flow of confirmation between these theories.

\subsection{Pre-'Reductive' Confirmation}

Let $\boldsymbol{P}_{1}(\mathrm{~S})$ and $\boldsymbol{P}_{1}(\mathrm{G})$ be the marginal probabilities of the root nodes $S$ and $G$ of the Bayesian network in Figure 7 , where $\boldsymbol{P}_{1}$ is the relevant probability measure. Let $\boldsymbol{P}_{1}(\mathrm{E} \mid \mathrm{G})$ and $\boldsymbol{P}_{1}(\mathrm{E} \mid \neg \mathrm{G})$ be the conditional probabilities of the child node $E$. For convenience, we use the following abbreviation scheme:

$$
\begin{aligned}
\boldsymbol{P}_{1}(\mathrm{~S}) & =\sigma, \boldsymbol{P}_{1}(\mathrm{G})=\gamma, \\
\boldsymbol{P}_{1}(\mathrm{E} \mid \mathrm{G}) & =\pi, \boldsymbol{P}_{1}(\mathrm{E} \mid \neg \mathrm{G})=\rho
\end{aligned}
$$

We assume a positive confirmatory relation between $E$ and $G$, such that $\pi>\rho$.

From the network structure in Figure 7, we can read off the conditional and unconditional independencies $E \Perp S \mid G$ and $S \Perp G$, such that $\boldsymbol{P}_{1}(\mathrm{~S} \mid \mathrm{E})=\boldsymbol{P}_{1}(\mathrm{~S})$. Evidence $\mathrm{E}$ does not confirm (or disconfirm) S. Hence, there is no flow of confirmation from the syntactic to the semantic theory. In the absence of the relation $r: \mathbb{S} \rightarrow \mathbb{G}$, the variables $S$ and $G$ are probabilistically independent before the establishment of their relation. This fact is captured by equation (3):

$$
\boldsymbol{P}_{1}(\mathrm{~S}, \mathrm{G})=\boldsymbol{P}_{1}(\mathrm{~S}) \boldsymbol{P}_{1}(\mathrm{G})=\gamma \sigma
$$


By (3), the prior probability of the conjunction of S and G equals the product of the marginal probabilities of the positive instantiations of the root nodes. Using the methodology from [5], we obtain the posterior probability of the conjunction of $\mathrm{S}$ and $\mathrm{G}$ as follows:

$$
\boldsymbol{P}_{1}^{*}:=\frac{\boldsymbol{P}_{1}(\mathrm{~S}, \mathrm{G}, \mathrm{E})}{\boldsymbol{P}_{1}(\mathrm{E})}=\frac{\boldsymbol{P}_{1}(\mathrm{~S}, \mathrm{G}, \mathrm{E})}{\sum_{S, G}(S, G, \mathrm{E})}=\frac{\gamma \pi \sigma}{\gamma \pi+\bar{\gamma} \rho}
$$

The denominator of the rightmost fraction in (4) is a convex combination of $\pi$ and $\rho$ weighed by $\gamma$, where $\bar{\gamma}:=1-\gamma \cdot{ }^{30}$

We close the present subsection by assessing the degree of confirmation of the conjunction of $\mathrm{S}$ and $\mathrm{G}$. To do this, we use the difference measure $d$ (see [7]) which is defined for our case as follows: ${ }^{31}$

$$
d_{1}:=\boldsymbol{P}_{1}(\mathrm{~S}, \mathrm{G} \mid \mathrm{E})-\boldsymbol{P}_{1}(\mathrm{~S}, \mathrm{G})
$$

Thus, E confirms $\mathrm{G}$ if the consideration of E raises the probability of the conjunction of $\mathrm{S}$ and $\mathrm{G}$. By calculating $d_{1}$, we show that this is indeed the case:

$$
d_{1}=\frac{\gamma \bar{\gamma} \sigma(\pi-\rho)}{\gamma \pi+\bar{\gamma} \rho}
$$

If we assume that $\gamma, \pi, \rho$, and $\sigma$ lie in the open interval $(0,1)$, where $\pi>\rho$, the above fraction is always strictly positive. We summarize our observation in the following proposition:

Proposition 1 E confirms $S$ and $G$ iff $E$ confirms $G$.

This completes our investigation of the joint probability of $\mathrm{S}$ and $\mathrm{G}$ before Montague's relation of $\mathrm{G}$ to $\mathrm{S}$. We next investigate the joint probability of $\mathrm{S}$ and $\mathrm{G}$ after such a relation has been established.

\subsection{Post-'Reductive' Confirmation}

To determine the confirmation of $\mathrm{S}$ and $\mathrm{G}$ in the post-'reductive' situation (see Fig. 8), we must first restate the probability distributions from the previous subsection. In particular, since $G$ is no longer a root node in Figure 8 (and is, thus, not assigned a prior probability), we replace the equation $\boldsymbol{P}_{1}(\mathrm{G})=\gamma$ from (2) by the equations from (7), where $\boldsymbol{P}_{2}$ is the new probability measure:

$$
\boldsymbol{P}_{2}(\mathrm{G} \mid \mathrm{S})=1, \boldsymbol{P}_{2}(\mathrm{G} \mid \neg \mathrm{S})=0
$$

The equations from (7) are warranted by Montague's relation $r$. All other assignments are as for $\boldsymbol{P}_{1}$. Our introduction of the new measure $\boldsymbol{P}_{2}$ is motivated by the move to a different probabilistic situation and by the need to assign

\footnotetext{
30 We will hereafter abbreviate ' $1-x$ ' as ' $\bar{x}$ '.

31 As is discussed in [19] (see [18]), results may depend on our choice of confirmation measure. Whether (and to what extent) they do, will be a question for future research.
} 
to the received Montagovian propositions possibly distinct probabilistic values. Equality statements of the form $\boldsymbol{P}_{2}(\mathrm{~S})=\boldsymbol{P}_{1}(\mathrm{~S})$ ensure the possibility of comparing the confirmation of $\mathrm{S}$ and $\mathrm{G}$ in the two situations.

As is encoded by the arrow from $S$ to $G$ in Figure 8, Montague's relation $r$ effects a flow of evidence from syntax to semantics. The confirmation of $\mathrm{S}$ is defined simply as follows:

Proposition $2 E$ confirms $S$ iff $\pi>\rho$.

According to the above proposition, the evidence $\mathrm{E}$ confirms the proposition $\mathrm{S}$ if (as has been assumed in Section 4.1) E supports G. The equations in (7) ensure a positive flow of confirmation from $\mathrm{G}$ to $\mathrm{S}$.

On the basis of the above, the conjunction of $\mathrm{S}$ and $\mathrm{G}$ has the following prior and posterior probabilities: (All calculations are included in the Appendix.)

$$
\begin{aligned}
\boldsymbol{P}_{2}(\mathrm{~S}, \mathrm{G}) & =\sigma \\
\boldsymbol{P}_{2}^{*}:=\boldsymbol{P}_{2}(\mathrm{~S}, \mathrm{G} \mid \mathrm{E}) & =\frac{\pi \sigma}{\pi \sigma+\rho \bar{\sigma}}
\end{aligned}
$$

The degree of confirmation of the conjunction of S and G under the measure $\boldsymbol{P}_{2}$ is recorded below:

$$
d_{2}:=\boldsymbol{P}_{2}(\mathrm{~S}, \mathrm{G} \mid \mathrm{E})-\boldsymbol{P}_{2}(\mathrm{~S}, \mathrm{G})=\frac{\sigma \bar{\sigma}(\pi-\rho)}{\pi \sigma+\rho \bar{\sigma}}
$$

This completes our investigation of the probabilities and confirmation of the conjunction of S and G in the post-'reductive' situation. To show the epistemic value of our Montagovian model of intertheoretic relations, we next compare the conjunction's probabilities and confirmation in the two scenarios. We accept the establishment of an intertheoretic relation if it raises the conjunction's probabilities or evidential support, and reject (or ignore) it otherwise.

\subsection{Comparing Situations}

We begin by comparing the prior probabilities of the conjunction of S and G in the two situations from Sections 4.1 and 4.2. While the propositional variables $S$ and $G$ are independent before the establishment of their relation, they have become dependent after the establishment of this relation. This is due to the fact that $G$ is no longer a root node in Figure 8. To compare the joint probabilities of $\mathrm{S}$ and $\mathrm{G}$ in the two scenarios, we assume the identity of $\boldsymbol{P}_{2}(\mathrm{G})$ and $\boldsymbol{P}_{1}(\mathrm{G})$, and of $\boldsymbol{P}_{2}(\mathrm{E} \mid \mathrm{G})$ and $\boldsymbol{P}_{1}(\mathrm{E} \mid \mathrm{G})$. By the first equality in (7), we further assume the equality in (11), such that $\gamma=\sigma$.

$$
\boldsymbol{P}_{2}(\mathrm{G})=\boldsymbol{P}_{2}(\mathrm{G} \mid \mathrm{S}) \boldsymbol{P}_{2}(\mathrm{~S})=\sigma
$$

Using the above, we calculate the difference, $\Delta_{0}$, between the conjunction's pre- and post-'reductive' prior probabilities, and obtain

$$
\Delta_{0}:=\boldsymbol{P}_{2}(\mathrm{~S}, \mathrm{G})-\boldsymbol{P}_{1}(\mathrm{~S}, \mathrm{G})=\sigma \bar{\sigma} .
$$


Intuitively, Montague's syntax-semantics relation is epistemically valuable if the prior probability of the conjunction of $\mathrm{S}$ and $\mathrm{G}$ is higher after the establishment of a relation between $\mathbb{S}_{\mathrm{S}}$ and $\mathbb{G}_{\mathrm{S}}$, i.e. if $\Delta_{0}>0$. Since we assume that all non- $r$-based probabilities are non-extreme, we know that the former is indeed the case.

The difference, $\Delta_{1}$, between the conjunction's posterior probabilities under the measures $\boldsymbol{P}_{2}$ and $\boldsymbol{P}_{1}$ is also strictly positive:

$$
\Delta_{1}:=\boldsymbol{P}_{2}(\mathrm{~S}, \mathrm{G} \mid \mathrm{E})-\boldsymbol{P}_{1}(\mathrm{~S}, \mathrm{G} \mid \mathrm{E})=\frac{\pi \sigma \bar{\sigma}}{\pi \sigma+\rho \bar{\sigma}}
$$

To show the truth of this statement, we use the above assumptions together with the fact that $\pi>\rho$.

The post-'reductive' confirmation of our propositions witnesses a similar increase. To establish this, we calculate the difference between the conjunction's pre- and post-'reductive' degree of confirmation under the difference measure, and obtain

$$
\Delta_{2}:=d_{2}-d_{1}=\frac{\sigma \bar{\sigma}^{2}(\pi-\rho)}{\pi \sigma+\rho \bar{\sigma}} .
$$

As can be read off from the expression in (14), the positivity of $\Delta_{2}$ - and the attendant positive confirmatory impact of Montague's syntax-semantics relation - is conditional on the requirement that $\sigma \in(0,1)$ and that $\pi>\rho$.

The above-observed increase in the joint probabilities and evidential support of the conjunction of $\mathrm{S}$ and $\mathrm{G}$ corresponds to the increase in a conjunction's probabilities and support after the execution of a Nagelian reduction (see [15]). In particular, since Nagelian bridge laws and Montague's relation $r$ both set the posterior probability of the truth of the 'phenomenological' proposition (given the truth of the 'fundamental' proposition) to 1, Montague's relation between categorial grammar and model-theoretic semantics achieves an equally large ${ }^{32}$ boost in confirmation as the Nagelian reduction of a 'suitable"33 proposition-pair. This observation is captured below:

Observation For suitable pairs of propositions, the establishment of an intertheoretic relation along the lines of Montague's syntax-semantics relation is epistemically equally advantageous as Nagel Reduction.

\footnotetext{
32 To ensure the comparability of the post-'reductive' situation from Section 4.2 with the post-reductive situation from [15] - which assumes Schaffner's [52] revised model of Nagel Reduction (see Sect. 5.2) -, we let the posterior probability of the truth, $\mathrm{T}_{1}^{*}$, of the corrected version of the 'fundamental' proposition (given the truth, $\mathrm{T}_{1}$, or falsity, $\neg \mathrm{T}_{1}$, of the 'uncorrected' fundamental proposition) and the truth, $\mathrm{T}_{2}$, of the uncorrected version of the 'phenomenological' proposition (given the truth, $\mathrm{T}_{2}^{*}$, or falsity, $\neg \mathrm{T}_{2}^{*}$, of the corrected 'phenomenological' proposition) both be 1 (resp. 0).

33 Here, suitable is defined as 'allowing the application of the described procedure for the establishment of the relevant intertheoretic relation'. As a result, suitable propositions for Nagelian reduction have a common target domain and contain surjectively related predicates.
} 


\section{Future Work}

We close our paper by suggesting two possible extensions of our model of Montague's syntax-semantics relation that may be further developed in future work. These extensions include the adaptation of this model to fundamental theories with multiple propositions (in Sect. 5.1) and its adaptation to a variant of Schaffner's [52] revised model of Nagelian reduction (in Sect. 5.2). We will see that the latter accounts for the structural richness of grammatical rules and that it elucidates the relation of strong analogy between the original and the 'corrected' version of each proposition in the phenomenological theory.

\subsection{Extension I: Relations between 'Larger' Theories}

The previous section has shown that our Montagovian model of the syntaxsemantics relation increases the joint probabilities and degree of confirmation of the conjunction of pairs of theories with a single propositional element. Montague's relation $r$ from Section 2.2 suggests an easy generalization of this result to pairs with more comprehensive phenomenological theories (e.g. to syntactic theories with more categories of expressions, see Fig. 2) in which the behavior of the syntactic operations is governed by a larger set of rules. ${ }^{34}$ The intertheoretic relation between these 'larger' theories is represented by the graph from Figure 9. In the graph, we call the variables $S, G$, and $E$ from Section 4 ' $S_{1}$ ', ' $G_{1}$ ', and ' $E_{1}$ ', respectively. The variables $S_{i}, G_{i}$, and $E_{i}$ (with $1<$ $i \leq n \in \mathbb{N}$ ) are associated with new (semantic or syntactic) rules and their supporting pieces of evidence.

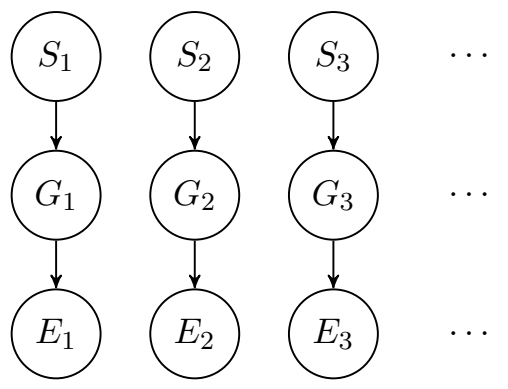

Fig. 9 Post-'reductive' relations between pairs of propositions $\left\langle S_{k}, G_{k}\right\rangle$.

As is captured in Figure 9, Montague's relation $r$ effects a pairwise relation between the above rules, which relates categorial grammar to model-theoretic semantics by relating $\mathbb{G}_{1}$ to $\mathbb{S}_{1}, \mathbb{G}_{2}$ to $\mathbb{S}_{2}, \mathbb{G}_{3}$ to $\mathbb{S}_{3}$, etc. As a result, the proba-

\footnotetext{
34 The (syntactic and semantic) rules for the behavior of common nouns (see fn. 14.) are examples of such new rules.
} 
bility of linguistic syntax 'related' to semantics will correspond to the product of the probabilities of all individual proposition-pairs:

$$
\begin{aligned}
\boldsymbol{P}_{2}\left(\bigcap_{k}\left\langle\mathrm{~S}_{k}, \mathrm{G}_{k}\right\rangle\right) & =\boldsymbol{P}_{2}\left(\mathrm{~S}_{1}, \mathrm{G}_{1}\right) \boldsymbol{P}_{2}\left(\mathrm{~S}_{2}, \mathrm{G}_{2}\right) \boldsymbol{P}_{2}\left(\mathrm{~S}_{3}, \mathrm{G}_{3}\right) \ldots \\
& =\boldsymbol{P}_{2}\left(\mathrm{~S}_{1}\right) \boldsymbol{P}_{2}\left(\mathrm{~S}_{2}\right) \boldsymbol{P}_{2}\left(\mathrm{~S}_{3}\right) \ldots
\end{aligned}
$$

respectively

$$
\begin{aligned}
\boldsymbol{P}_{2}\left(\bigcap_{k}\left\langle\mathrm{~S}_{k}, \mathrm{G}_{k} \mid \mathrm{E}_{k}\right\rangle\right) & =\boldsymbol{P}_{2}\left(\mathrm{~S}_{1}, \mathrm{G}_{1} \mid \mathrm{E}_{1}\right) \boldsymbol{P}_{2}\left(\mathrm{~S}_{2}, \mathrm{G}_{2} \mid \mathrm{E}_{2}\right) \boldsymbol{P}_{2}\left(\mathrm{~S}_{3}, \mathrm{G}_{3} \mid \mathrm{E}_{3}\right) \ldots \\
& =\boldsymbol{P}_{2}\left(\mathrm{~S}_{1} \mid \mathrm{E}_{1}\right) \boldsymbol{P}_{2}\left(\mathrm{~S}_{2} \mid \mathrm{E}_{2}\right) \boldsymbol{P}_{2}\left(\mathrm{~S}_{3} \mid \mathrm{E}_{3}\right) \ldots
\end{aligned}
$$

However, the stipulation of independent morphisms between all pairs $\left\langle S_{k}, G_{k}\right\rangle$ does not assign to the linguistic syntax-semantics relation an optimal epistemic value. This is due to the multiplication properties of real numbers in the open interval $(0,1)$, such that the probability of the conjunction decreases in inverse proportion to the number of its conjuncts. But this contradicts our intuition (reflected in much work in formal semantics and in [15, p. 326]) that the establishment of relations between 'larger' (multi-proposition) theories shares the epistemological advantages of the establishment of relations between 'smaller' theories.

Admittedly, the observed decrease in the joint probabilities of larger theories is also a problem for Nagelian reduction. However, Montague-style semantics provides a strategy for avoiding this problem. This strategy is based on the possibility of constructing (the objects in) certain semantic domains from (the objects in) other domains: ${ }^{35}$ Our presentation of categorial grammar and model-theoretic semantics from Section 2.1 has assumed that all semantic domains (especially the domains $\mathcal{D}_{\mathrm{N}}, \mathcal{D}_{\mathrm{V}}$, and $\mathcal{D}_{\mathrm{N}}$ ) are equally basic. However, in Montague-style frameworks, this is in general not the case. In particular, to enable the compositional interpretation of natural language, Montagovian models only contain basic domains for individuals (i.e. the set $\mathcal{D}_{\mathrm{N}}$ ) and truth-values (i.e. the set $\mathcal{D}_{\mathrm{S}}$ ) (see [37-39]). From the elements of these domains, elements of all other domains (e.g. the members of the set $\mathcal{D}_{\mathrm{V}}$ ) are obtained via semantic operations like function-space formation (s.t. $\mathcal{D}_{\mathrm{V}} \subseteq\left\{f \mid f:\left(\mathcal{D}_{\mathrm{N}} \rightarrow \mathcal{D}_{\mathrm{S}}\right)\right\}$ ).

We expect that these constructibility relations between domains will establish connections between objects of the fundamental theory (and similarly, for the phenomenological theory) which result in mutual probabilistic dependencies between same-theory propositions. A full development of this sophisticated variant of our Montagovian model of intertheoretic relations, and an assessment of its epistemological merits, will be provided in a sequel to this paper.

35 A similar observation applies to syntactic categories (see [1]). 


\subsection{Extension II: Schaffner-Style Relations}

Section 2.2 has identified our model of Montague's syntax-semantics relation as a model of a weak type of intertheoretic relation. We have attributed this weakness to the fact that the syntactic rule $\mathbb{G}_{\mathrm{S}}$ contains more structural information than the semantic rule $\mathbb{S}_{\mathrm{s}}$. We have used this fact to argue for the lower reductive strength of Montague's syntax-semantics relation vis-à-vis Nagelian reduction.

Notably, however, Nagelian reduction also does not enable the full reduction of phenomenological to fundamental propositions. For example, in the reduction of thermodynamics to statistical mechanics, Nagel's model is unable to derive the exact Second Law of thermodynamics, in which entropy does not fluctuate in equilibrium. ${ }^{36}$

Schaffner's revised model of Nagelian reduction from [52, 53] (see [42]) improves upon the reductive accuracy of the Nagelian model. To do this, it introduces a dedicated level of 'corrected' propositions of the two theories and demands that the corrected version of each proposition of the fundamental theory be obtained from the original proposition via auxiliary assumptions and that the corrected version of each proposition of the phenomenological theory be strongly analogous to the original proposition. ${ }^{37}$ The resulting model is captured in Figure 10. In the figure, $T_{1}^{*}$ and $T_{2}^{*}$ are the 'corrected' versions of the propositions $T_{1}$ and $T_{2}$, respectively.

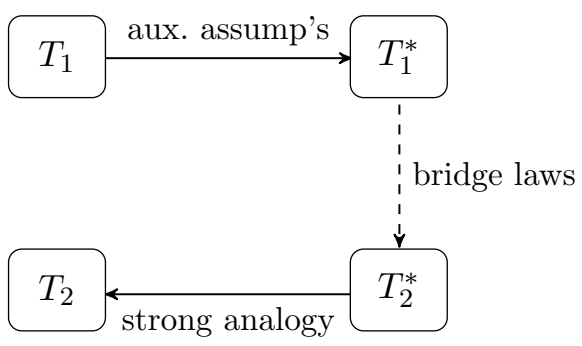

Fig. 10 Generalized Nagel-Schaffner reduction.

We expect that the introduction of a level of corrected propositions will also improve the success of our model of Montague's syntax-semantics relation. In Section 2.2, we have already noted that the syntactic correlate of function application will not contain any information about word order. We have attributed this observation to the fact that semantics contains much less structural information than syntax. However, since word order is a very stable property of a language, the specification of a language's word order-type will allow us to supplement this information. In particular, since strong analogy is

\footnotetext{
36 Nagelian reduction can only derive a variant of this law, in which thermodynamic entropy does fluctuate in equilibrium.

37 For a detailed presentation and discussion of generalized Nagel-Schaffner reduction, the reader is referred to [52] and [14].
} 
a contextual relation, we can say that $\{Y X\}$ is only strongly analogous to its order-sensitive variant $[X Y]$ in the context of subject-verb-object (SVO) languages. This strong analogy will allow us to 'adjust' the rule from (1b) to the syntactic rule from (2). The latter describes the combination of intransitive verbs with a name which occurs on their left.

(2) If $Y \in \mathcal{E}_{\mathrm{V}}$ and $X \in \mathcal{E}_{\mathrm{N}}$, then $[X Y] \in \mathcal{E}_{\mathrm{S}}$.

Since the rule (1b) is equivalent to the rule (2) in SVO-contexts, a Schaffnerstyle variant of our Montagovian model will derive the 'right' concatenation rule for proper names and intransitive verbs.

Apart from accounting for the structural richness of syntactic rules, the supplementation of information about a language's word-order type helps elucidate Schaffner's 'strong analogy' relation: Since its introduction in [52], this relation has been criticized for its vagueness and context-dependency. These properties are witnessed by the fact that there is no general characterization of strong analogy and that what counts as strongly analogous differs from case to case. Our Schaffner-style variant of Montague's syntax-semantics relation answers some of the criticisms against strong analogy. In particular, it shows that, for a clearly delineated case (i.e. the relation between the rules (1b) and (2)), strong analogy is a well-defined relation ${ }^{38}$ which serves its intended purpose.

The elaboration of the described variant of our model of Montague's syntaxsemantics relation, and a demonstration of its (expected) epistemic advantages, is left for another occasion. We close the paper with a summary of our main results.

\section{Conclusion}

In this paper, we have presented a model of a new type of intertheoretic relation which is inspired by a Montague-style formal semantic framework for the analysis and interpretation of natural language syntax. We have identified the commonalities of our Montagovian model of the syntax-semantics relation with the classical model of Nagelian reduction and have established their salient differences. In particular, we have observed that our model of Montague's syntax-semantics relation can capture directed dependency relations between pairs of theories with non-overlapping target domains, which cannot be captured by the Nagelian model. To show the epistemic rationale behind our new type of intertheoretic relation, we have demonstrated that - like its Nagelian counterpart - the Montagovian relation raises the posterior probability of the conjunction of the two related theories and increases the flow of confirmation between them. Finally, we have identified two strategies for the extension and improvement of our model of Montague's syntax-semantics relation.

\footnotetext{
38 Thus, (a) is strongly analogous to (b) if all members of $\mathcal{E}$ which are mentioned in (b) belong to an SVO-language and if (a) is the result of replacing all occurrences of $\{Y X\}$ by occurrences of $[X Y]$.
} 
Appendix: Proofs and Calculations for Section 4

We have calculated the pre-'reductive' probabilities of the conjunction of the positive instantiations of $S$ and $G$ in Section 4.1. The joint distribution, $\boldsymbol{P}_{2}(S, G, E)$, of the (post-'reductive') graph in Figure 8 is given by the expression

$$
\boldsymbol{P}_{2}(S) \boldsymbol{P}_{2}(G) \boldsymbol{P}_{2}(E \mid G) .
$$

Using the methodology from [5], the prior probability of the conjunction of $\mathrm{S}$ and $\mathrm{G}$ is obtained as follows:

$$
\boldsymbol{P}_{2}(\mathrm{~S}, \mathrm{G})=\sum_{E} \boldsymbol{P}_{2}(\mathrm{~S}, \mathrm{G}, E)=\pi \sigma+\bar{\pi} \sigma=\sigma
$$

We yield the posterior probability, $\boldsymbol{P}_{2}^{*}:=\boldsymbol{P}_{2}(\mathrm{~S}, \mathrm{G} \mid \mathrm{E})$, of the conjunction of $\mathrm{S}$ and $\mathrm{G}$ thus:

$$
\boldsymbol{P}_{2}^{*}=\frac{\boldsymbol{P}_{2}(\mathrm{~S}, \mathrm{G}, \mathrm{E})}{\boldsymbol{P}_{2}(\mathrm{E})}=\frac{\pi \sigma}{\pi \sigma+\rho \bar{\sigma}}
$$

To obtain the difference $\Delta_{0}$, we calculate

$$
\boldsymbol{P}_{2}(\mathrm{~S}, \mathrm{G})-\boldsymbol{P}_{1}(\mathrm{~S}, \mathrm{G})=\sigma-\sigma^{2}=\sigma \bar{\sigma} .
$$

This proves the following proposition:

Proposition $3 \Delta_{0}=0$ iff $\sigma=0$ or $1 ; \Delta_{0}>0$ iff $\sigma \in(0,1)$.

The difference, $\Delta_{1}$, between the conjunction's pre- and post-'reductive' posterior probabilities is obtained as follows:

$$
\Delta_{1}:=\boldsymbol{P}_{2}^{*}-\boldsymbol{P}_{1}^{*}=\frac{\pi \sigma-\pi \sigma^{2}}{\pi \sigma+\rho \bar{\sigma}}=\frac{\pi \sigma \bar{\sigma}}{\pi \sigma+\rho \bar{\sigma}}
$$

From the difference measure

$$
d_{2}:=\boldsymbol{P}_{2}(\mathrm{~S}, \mathrm{G} \mid \mathrm{E})-\boldsymbol{P}_{2}(\mathrm{~S}, \mathrm{G})=\frac{\sigma \bar{\sigma}(\pi-\rho)}{\pi \sigma+\rho \bar{\sigma}},
$$

we calculate the difference, $\Delta_{2}$, between the conjunction's degree of confirmation before and after the establishment of Montague's syntax-semantics relation as follows:

$$
\Delta_{2}:=d_{2}-d_{1}=\frac{\sigma \bar{\sigma}(\pi-\rho)-\sigma^{2} \bar{\sigma}(\pi-\rho)}{\pi \sigma+\rho \bar{\sigma}}=\frac{\sigma \bar{\sigma}^{2}(\pi-\rho)}{\pi \sigma+\rho \bar{\sigma}}
$$

This completes our proofs and calculations for Section 4.

Acknowledgements We wish to thank Lucas Champollion, Paul Egré, Branden Fitelson, Greg Novack, Peter Pagin, Jan Sprenger, Markus Werning, Rineke Verbrugge, and three anonymous reviewers for JoLLI for valuable input about the content of this paper. We owe our audiences at the Future of Philosophy of Science Conference, FEW 11, Bridges 2014, and the Tokyo Forum for Analytic Philosophy for many helpful comments and suggestions. The research for this paper is supported by Tilburg University (via K. Liefke's doctoral fellowship), the Alexander von Humboldt Foundation (via S. Hartmann's Humboldt Professorship), and the German Research Foundation (via K. Liefke's grant LI 2562/1-1). 


\section{References}

[1] Kazimierz Ajdukiewicz, Die syntaktische Konnexität, Studia Philosophica 1 (1935), 1-27.

[2] Emmon Bach, An extension of classical transformational grammar, Problems of Linguistic Metatheory: Proceedings of the 1976 conference (R. Saenz, ed.), Michigan State University, Michigan, 1976, pp. 183-224.

[3] Renate Bartsch and Theo Vennemann, Semantic Structures, Athenäum, Frankfurt, a.M., 1972.

[4] John Bickle, Reducing mind to molecular pathways: explicating the reductionism implicit in current cellular and molecular neuroscience, Synthese (2006), 411-434.

[5] Luc Bovens and Stephan Hartmann, Bayesian Epistemology, Clarendon Press, Oxford, 2003.

[6] Rudolf Carnap, Logische Syntax der Sprache, Springer, New York, 1968.

[7] _ Empiricism, semantics, and ontology, Revue Internationale de Philosophie 4 (1950), 20-40; reprinted in Meaning and Necessity: a study in semantics and modal logic, The University of Chicago Press, Chicago and London, 1988.

[8] Gennaro Chierchia and Raymond Turner, Semantics and property theory, Linguistics and Philosophy 11/3 (1988), 261-302.

[9] Noam Chomsky, Deep structure, surface structure, and semantic representation, Semantics: an interdisciplinary reader (D. Steinberg and L. Jakobovits, eds.), Cambridge University Press, Cambridge, 1971.

[10] _ Linguistics and brain science, Image, Language, Brain (Alec Marantz, Yasushi Miyashita, and Wayne O'Neil, eds.), MIT Press, Cambridge, Mass., 2000.

[11] Alonzo Church, A formulation of the Simple Theory of Types, Journal of Symbolic Logic 5/2 (December 1940), 56-68.

[12] Maxwell J. Cresswell, Logics and Languages, Methuen Young Books, London, 1973.

[13] Lindley Darden and Nancy Maull, Interfield theories, Philosophy of Science 44/1 (1977), 43-64.

[14] Foad Dizadji-Bahmani, Roman Frigg, and Stephan Hartmann, Who's afraid of Nagelian reduction?, Erkenntnis 73/3 (2010), 393-412.

[15] _ Confirmation and reduction: a Bayesian account, Synthese 179/2 (2011), 321-338.

[16] John Earman, Bayes or Bust?, The MIT Press, Cambridge, Mass., 1992.

[17] Rolf A. Eberle, Replacing One Theory by Another under Preservation of a Given Feature, Philosophy of Science 38/4 (December 1971), 486-501.

[18] Ellery Eells and Branden Fitelson, Measuring confirmation and evidence, The Journal of Philosophy 97/12 (December 2000), 663-672.

[19] Branden Fitelson, The plurality of Bayesian measures of confirmation and the problem of measure sensitivity, Philosophy of Science 66 (1999), 362378. 
[20] Graeme Forbes, Content and theme in attitude ascriptions, NonPropositional Intentionality (Alex Grzankowski and Michelle Montague, eds.), Oxford University Press, Oxford and New York, Forthcoming.

[21] Alan Hájek and Stephan Hartmann, Bayesian epistemology, A Companion to Epistemology (Jonathan Dancy, Ernest Sosa, and Matthias Steup, eds.), Blackwell Companions to Philosophy, Blackwell, Malden, Mass., 2010.

[22] Jeremy Hartman, Varieties of Clausal Complementation, Doctoral dissertation, Massachusetts Institute of Technology, Cambridge, Mass., 2012.

[23] Stephan Hartmann, Models and stories in hadron physics, Models as Mediators (Mary S. Morgan and Margaret Morrison, eds.), Cambridge University Press, Cambridge, 1999, pp. 326-346.

[24] Stephan Hartmann and Jan Sprenger, Bayesian epistemology, Routledge Companion to Epistemology (Sven Bernecker and Duncan Pritchard, eds.), Routledge, Malden, Mass., 2010.

[25] Carl Gustav Hempel, Philosophy of Natural Science, Prentice-Hall, Englewood Cliffs, 1966.

[26] Herman Hendriks, Flexible Montague Grammar, ITLI Prepublication Series for Logic, Semantics and Philosophy of Language 08 (1990).

[27] _ Studied Flexibility: Categories and types in syntax and semantics, Doctoral dissertation, Institute for Logic, Language and Computation, Amsterdam, 1993.

[28] Kevin D. Hoover, Idealizing reduction: the microfoundations of macroeconomics, Erkenntnis 73/3 (2010), 329-347.

[29] Colin Howson and Peter Urbach, Scientific Reasoning: the Bayesian approach, Open Court, La Salle, 2005.

[30] Itamar Kastner, Factivity mirrors interpretation: The selectional requirements of presuppositional verbs, Lingua 164, Part A (2015), 156-188.

[31] John G. Kemeny and Paul Oppenheim, On reduction, Philosophical Studies 19 (1967), 6-19.

[32] Theo A.F. Kuipers, The reduction of phenomenological to kinetic thermostatics, Philosophy of Science 49/1 (March 1982), 107-119.

[33] Richard Levins and Richard Lewontin, Dialectics and reductionism in ecology, Synthese 43 (1980), 47-78.

[34] David Lewis, General semantics, Synthese 22/1-2 (December 1970), 18 67.

[35] Lia Litosseliti (ed.), Research Methods in Linguistics, Continuum, London and New York, 2010.

[36] Sandra D. Mitchell, Biological Complexity and Integrative Pluralism, Cambridge University Press, Cambridge and New York, 2003.

[37] Richard Montague, English as a formal language, Formal Philosophy: selected papers of Richard Montague (Richmond H. Thomason, ed.), Yale University Press, New Haven and London, 1976.

[38] _ Universal grammar, Formal Philosophy: selected papers of Richard Montague (Richmond H. Thomason, ed.), Yale University Press, New Haven and London, 1976. 
[39] _ The proper treatment of quantification in ordinary English, Formal Philosophy: selected papers of Richard Montague (Richmond H. Thomason, ed.), Yale University Press, New Haven and London, 1976.

[40] Michael Moortgat, Categorial type logics, Handbook of Logic and Language (Johan van Benthem and Alice G.B. ter Meulen, eds.), Elsevier Science Publishers, Amsterdam, 1997.

[41] Ernest Nagel, The Structure of Science, Routledge and Kegan Paul, London, 1961.

[42] _ , Teleology revisited, The Journal of Philosophy 84 (1977), 261301.

[43] Richard Neapolitan, Learning Bayesian Networks, Prentice Hall, 2003.

[44] Barbara Partee, Noun phrase interpretation and type-shifting principles, Studies in Discourse Representation Theory and the Theory of Generalized Quantifiers (Jeroen Groenendijk, Dick de Jong, and Martin Stokhof, eds.), Foris Publications, Dordrecht, 1987.

[45] _ Montague grammar, Handbook of Logic and Language: 2nd edition (Johan van Benthem and Alice G.B. ter Meulen, eds.), Elsevier Science Publishers, Amsterdam, 2011, 1997.

[46] Judea Pearl, Probabilistic Reasoning in Intelligent Systems: networks of plausible inference, Morgan Kauffman, San Francisco, 1988.

[47] Robert J. Podesva and Devyani Sharma (eds.), Research Methods in Linguistics, Cambridge University Press, Cambridge and New York, 2013.

[48] David Poeppel and David Embick, Defining the relation between linguistics and neuroscience, Twenty-First Century Psycholinguistics: four cornerstones (A. Cutler, ed.), Lawrence Erlbaum Associates, Mahwah, NJ:, 2005.

[49] Christopher Potts, The lexical semantics of parenthetical -as and appositive -which, Syntax 5/1 (April 2002), 55-88.

[50] Sebastian M. Rasinger, Quantitative Research in Linguistics: an introduction, Bloomsbury, London etc., 2013.

[51] Ivan Sag, Thomas Wasow, and Emily Bender, Syntactic Theory: A formal approach, CSLI Publications, Stanford, 2003.

[52] Kenneth F. Schaffner, Approaches to reduction, Philosophy of Science 34 (1967), 137-147.

[53] _ Reductionism in biology: prospects and problems, PSA: Proceedings of the Biennial Meeting of the Philosophy of Science Association 1974 (1974), 613-632.

[54] _ Discovery and Explanation in Biology and Medicine, Science and its Conceptual Foundations, The University of Chicago Press, Chicago, 1993.

[55] Maurice Schouten and Huib Looren de Jong (eds.), The Matter of the Mind: philosophical essays of psychology, neuroscience, and reduction, Blackwell, Oxford, 2012.

[56] Shoichi Takahashi, The hidden side of clausal complements, Natural Language and Linguistic Theory 28/2 (2010), 343-380.

[57] Alfred Tarski, Andrzej Mostowski, and Raphael Mitchel Robinson, Undecidable theories, North-Holland, Amsterdam, 1953. 
[58] Marcel Weber, Philosophy of Experimental Biology, Cambridge Studies in Philosophy and Biology, Cambridge University Press, Cambridge and New York, 2005. 